\title{
Fuzzy Image Processing Based Architecture for Contrast Enhancement in Diabetic Retinopathy Images
}

\author{
Dr. Basma MohammedKamal Younis ${ }^{1}$ and Dua'a Basman Younis ${ }^{2}$ \\ ${ }^{1,2}$ Department of Computer Technology Engineering, Engineering Technical College, Northern Technical University \\ Mosul, Iraq \\ 11r_bmk@ntu.edu.iq, 2duaa_basman@yahoo.com
}

\begin{abstract}
Diabetic retinopathy" is damage to retina denotes one of the problems of diabetes which is a significant reason for visual infirmity and blindness. A comprehensive and routine eye check is important to early detection and rapid treatment. This study proposes a hardware system that can enhance the contrast in the diabetic retinopathy eye fundus images as a first step in different eye disease diagnoses. The fuzzy histogram equalization technique is proposed to increases the local contrast of Diabetic Retinopathy Images. First, a histogram construction hardware architecture for different image processing purposes has been built then modified with fuzzy techniques to create fuzzy histogram equalization architecture, which is used to enhance the original images. Both architectures are designed using a finite-state machine (FSM) technique and programmed with VHDL programming language. The first one is implemented using two (Spartan 3E-XC3S500 and Xilinx Artix-7 XC7A100T) kits, while the second architecture is implemented using (Spartan 3E-XC3S500) kit. The system consists also of a modified video graphics array (VGA) port to display the input and resulted images with a proper resolution. All the hardware outputs are compared to that results produce from MatLab for verification and the resulted images are tested by PSNR, MSE, ENTROPY , and AMBE.
\end{abstract}

Keywords: Histogram Equlization, Fuzzy, contrast enhancement, Diabetic Retinopathy, FPGA.

\section{INTRODUCTION}

Diabetic retinopathy is a form of eye disease arising in diabetes over several years. Modest veins within the retina hurt, veins end blocked, defective and randomly developing. DR is asymptomatic, it does not obstruct vision until it reaches the point of achieving propel arrangement. A pre-treatment can reduce the risk of complete vision loss by 50 percent to prevent DR improvement. It affects the two eyes usually and often does not have an early warning signal. DR is classified in two levels as non-proliferative diabetic retinopathy (NDPR) and proliferative diabetic retinopathy (PDR) [1]. Fundus images used in this work is a posterior eye imaging. These images are captured using the fundus camera device [2]. On the other hand Histograms contain a large series of applications. They provide a perfect notion of the brightness contrast feature of a picture [3].

The purpose of this paper is to design and develop a hardware system using FPGA that can perform fuzzy techniques using fuzzy histogram equalization. Fuzzy techniques have been used for enhancing the contrast in the digital medical images. The suitability of the fuzzy technique will be tested and suggested for enhancing diabetic retinopathy images with color fundus pictures.

In 2007 Santiago Sanchez-Solano et al. introduced a design strategy to ease the implementation in programmable chip systems of embedded fuzzy controllers. Application architecture based on fieldprogrammable gate arrays is used to build controllers. The platform includes different hardware to incorporate fuzzy inference modules with an overall processor that enables hybrid devices/software programs to be accomplished. This paper uses the technique and key chain theory to achieve a control system for the solution of mobility activities in a stand-alone vehicle [4].

A fuzzy logic-based histogram equalization (FHE) introduced by V. Magudeeswaran and C.G.Ravichandran, in 2013 is proposed to improve image contrast. Two stages of FHE are involved. First, the fuzzy histogram is computed based on the fuzzy set theory to deal more closely with the imprecision of gray levels than classic crisp histograms. The second phase divides the fuzzy histogram into two sub-histograms based on the main picture's median value and equalizes them to maintain the picture's brightness. The results of experiments show that the proposed process can remove 
the washed presence and consequences of many approaches effectively [5].

Sarni Suhaila Rahim et al, (2015) provide a new automatic identification in eye fundus images of diabetic retinopathy and maculopathy by fuzzy image processing . The developer system involves image collection, preprocessing of images with a mixture of fuzzy techniques, extraction of features, and classification of the image through the use of multiple machine learning methods. The fuzzy image processing support system is used to help test of diabetic retinopathy and to decrease the burden of the examination group [6].

Also, in 2016 F. Memon et al, a new approach to implementing the histogram equalization algorithm in Field Programmable Gate Array (FPGA) is presented by Model-Based Design. The methods suggested help develop and design the model in a fairly short time via removing time-consuming and weary work owing to manually encoding. The MATLAB model of the histogram equalization is translated into the implementation of FPGA devices via HDL Coder. The design has been applied to the Xilinx Virtex5 FPGA and can operate at an estimated frequency of $183.733 \mathrm{MHz}$ by operating less than $5 \%$ of available devices with a total power consumption of 0.367 watts. Experimental results indicate that the proposed design methodology provides an equally comparable image in MATLAB output at full resolution, and generates $494 \times 335$ pixel output image at $0.9057 \mathrm{msec}$ vs. $10.845 \mathrm{sec}$ in MATLAB, much faster than the real-time video rate [7].

In the year 2017, C. Sugapriya presents a system, which mainly focuses on the Fuzzy logic systems in the digital processing of images. The main concern of this system lies in the fuzzy logic application in image processing with a summary of fuzzy's logic and digital image processing [8].

A new automatic contrast enhancement system is proposed and tested on the PMUI in 2019 by Shaima Ibraheem Jabbar et al. The main tool behind this method is a fuzzy technique, involving three stages: fuzzification, modification in the membership equation and defuzzification. An upper fuzzy entropy improves the parameters of membership functionality for PMUI. The results of the Fuzzy technology outperforms the results of another technique with 21 percent based on these evaluation methods [9].

The rest of the paper is organized as follows: section 2 illustrates the theoretical background of the fuzzy histogram equalization technique while section 3 describes the proposed hardware design and implementation and section 4 presents the analysis results extracted from the proposed algorithm and section 5 contains conclusion.

\section{FUZZY HISTOGRAM EQUALIZATION (FHE)}

The fuzzy histogram equalization method increases the local contrast of the original image in addition to maintaining the image brightness. The fuzzy histogram is calculated by (1). A "fuzzy histogram is an array of real numbers $h(i), i \in(0,1 \ldots, L-1), \mathrm{h}$ (i) implies the incidence frequency of gray levels around $i$. When the gray value $f(i, j)$ is known as a fuzzy number $\mu_{F(i, j)}$, the fuzzy histogram is calculated by

$$
\mathrm{F} \leftarrow \mathrm{h}(\mathrm{i})+\sum_{i} \sum_{j} \mu_{F(i, j)}
$$

Where $\mu_{F(i, j)}$ is the fuzzy membership function. Fuzzy statistics are much more capable of handling the inaccuracies of gray values than traditional crisp histograms and thus provide a smooth histogram. The FHE technique not only maintains the brightness of the picture but it also works towards improving the original picture's local contrast. There are two stages to the FHE. In the first stage, a fuzzy histogram based on a fuzzy set theory for managing inaccurate gray levels is calculated in comparison with the traditional crisp histograms. The second stage divides the fuzzy histogram into a two-sub histogram based on the median value of the original picture and then it equalizes to maintain the picture's brightness $[5,10]$. Figure 1 show the flowchart of FHE system.

\section{FUZZY IMAGE CONTRAST ENHANCEMENT SYSTEM DESIGN AND IMPLEMENTATION}

Figure 2 shows a block diagram of the overall designed system, which contain three main parts; data acquirement, Fuzzy histogram equalization and display units. Details of each part will be described in the next sections.

\subsection{Data Acquirement Unit}

First fundus images database were collected and stored in Hard Disk Drive. Before reading the image by FPGA, the color picture file is read from a computer hard disk drive with the MatLab program, then the picture is transformed to a gray level by a simple specific statement with other operation in Matlab program then the resultant picture is saved in a .coe file and stored in a Block RAM 
that is generated by ISE IP core generator. The block diagram for this part is shown in figure 3. VHDL is used to generate the dual-port BRAM.

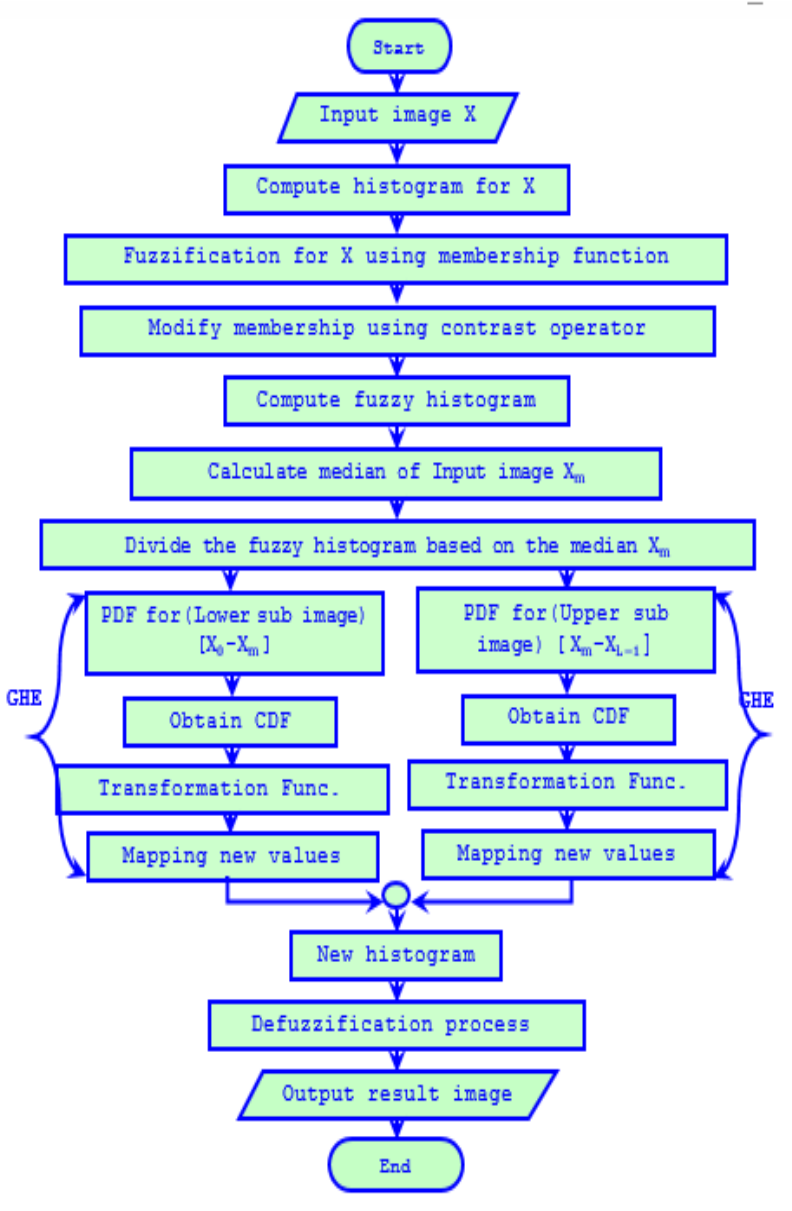

Fig. 1 Flowchart of FHE

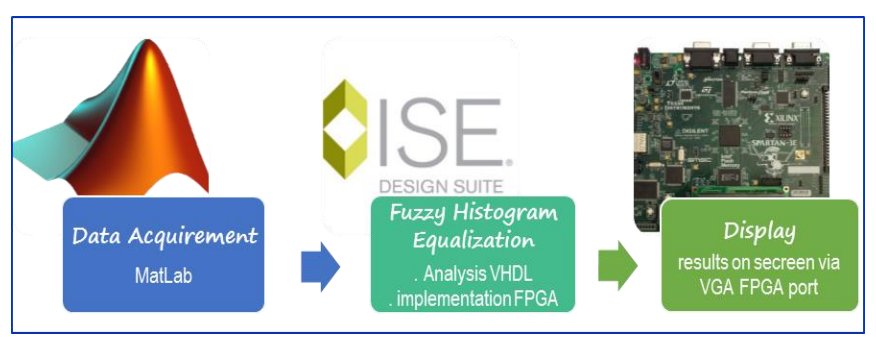

Fig.2. The Overall Hardware Designed System

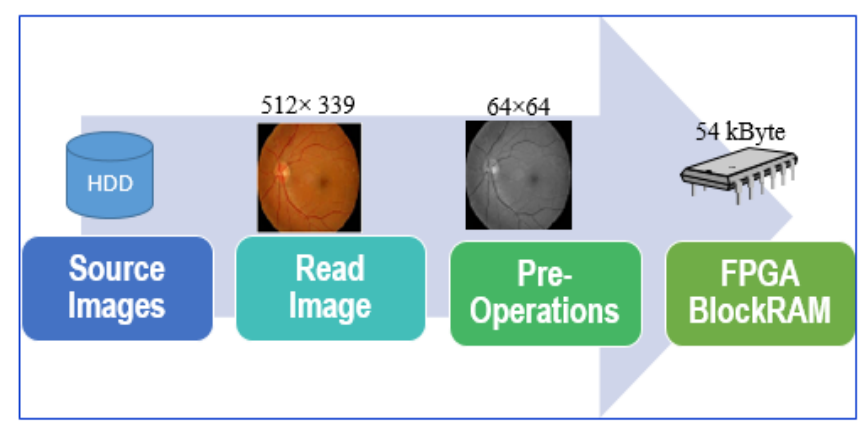

Fig. 3. Data Acquirement Unit Block Diagram

\subsection{Fuzzy Histogram Equalization Unit}

The block diagram for the fuzzy histogram equalization architecture is shown in figure 4. It consists of fuzzification unit, fuzzy membership modification unit, fuzzy histogram unit and defuzzification.

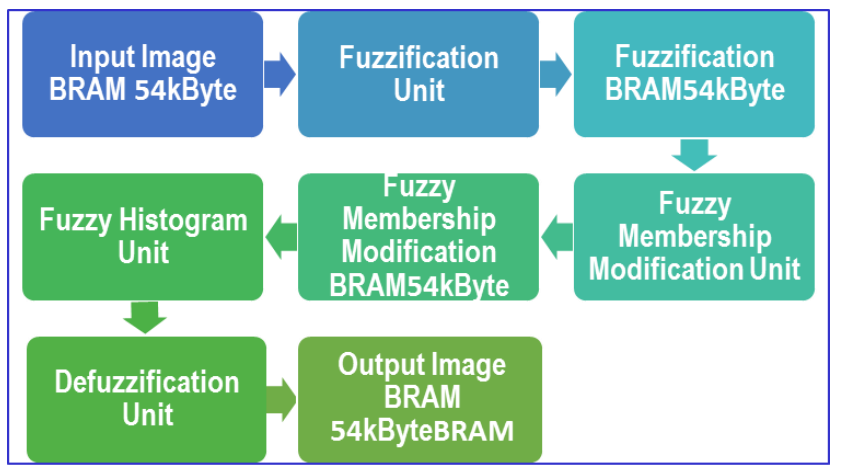

Fig. 4. Fuzzy Histogram Equalization Unit Block Diagram

\subsection{Display Unit}

The Spartan3E startup kit FPGA Starter involves a VGA display port via a DB15 adapter. As this port is connected directly by standard screen cable to most computer monitors or flat LCD screens [11]. Xilinx Spartan-3E XC3S500E of FPGA contain a 3-bits VGA display port which support only 8 colors to display on LCD screen for this reason an extension card is used to expand the VGA port to 6-bit to enhance the colors of the image displayed.

\section{ANALYSIS RESULTS}

A fuzzy histogram equalization system presented in this paper has been successfully tested as confirmed by the results in the following sections. These results include the performance of fuzzy image processing operations based on the fuzzy histogram equalization algorithm and display the enhanced images on LCD using FPGA device and with MatLab. The differences between the designs 
are also presented with their timing analysis, speedup, and performance metrics ...etc..

\subsection{Performance Evaluation}

The speed of execution or processing time is one of the basic characteristics of different algorithms in image processing, which is a measure of the running speed. The other important measurement parameter of the performance on the FPGA kit used is chip area occupation which measures the complexity of the design. The speed up can also be measured to compare hardware and software results, and shows how the hardware results are faster than the software one. Also the MSE, PSNR, $A M B E$ and entropy functions are used to calculate the quality of resulted image.

\subsection{Analysis Tools}

To obtain system performance analysis for algorithms in FPGA, different types of simulators and debugging analyzers are used such as ISim of ISE 10.1 and 14.7. In MATLAB, the TIC\&TOC function is used for time measuring to calculate the algorithms performance by putting it before and after codes needed to measure its execution time.

\subsection{Performance Analysis of the System}

First, the performance metrices for FHE enhancement techniqes is measured for sample images used in test (healthy, ModerateDR and severeDR) using MatLab. These performances is shown in table 1 for FHE results for $64 \times 64$ image sizes.

Table 1: FHE results for images of size $(64 \times 64)$ using MatLab

\begin{tabular}{|l|c|c|c|c|}
\hline image name & AMBE & MSE & PSNR & Entropy \\
\hline Healthy & 0.7719 & 8.5830 & 38.7944 & 6.1481 \\
\hline Moderate DR & 3.3972 & 51.8937 & 30.9796 & 5.7268 \\
\hline Severe DR & 3.8107 & 62.5197 & 30.1706 & 6.3684 \\
\hline
\end{tabular}

Second; the performance metrices for FHE enhancement techniqes is measured for the same sample images used in test using SPARTAN3E XC3S500E FPGA. These performances are shown in table 2. The performances metrics of FHE images using MatLab as shown in table 1 appeared better than those performances metrics of FHE images resulted using SPARTAN3E XC3S500E FPGA as shown in table 4.6 because the resolution of fixed-point numbers in VHDL is limited as compared with MatLab, also the hardware images are captured by the camera from LCD screen then it is used in the calculation of performances metrics in MatLab, as a result, the resolution of performances metrics of these images decreased.
Table 2: FHE results for images of size $(64 \times 64)$ using FPGA

\begin{tabular}{|l|c|c|c|c|}
\hline Image name & AMBE & MSE & PSNR & Entropy \\
\hline Healthy & 39.9621 & 1934.34 & 15.2654 & 6.1343 \\
\hline Moderate DR & 72.0654 & 5860.01 & 10.4518 & 6.3016 \\
\hline Severe DR & 52.5051 & 3495.58 & 12.6956 & 6.5152 \\
\hline
\end{tabular}

The following figures $(5,6,7,8,9$ and 10) illustrates the samples images and there results after the FHE using MatLab and FPGA.

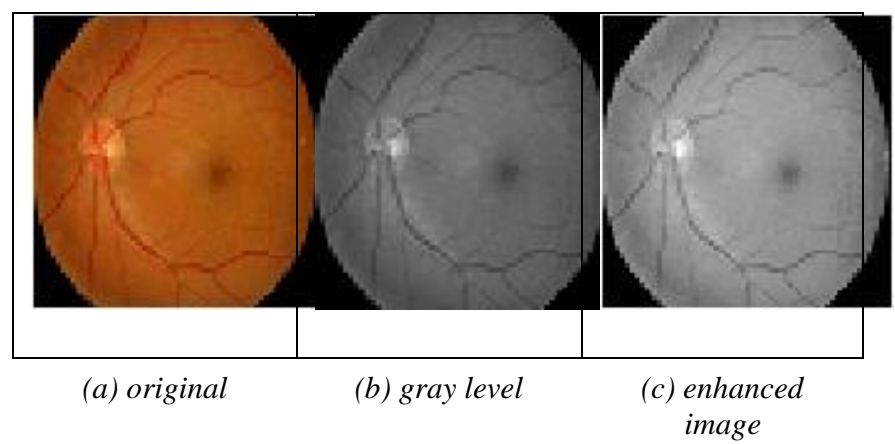

Fig. 5. MatLab result for healthy image with $64 \times 64$ resolution

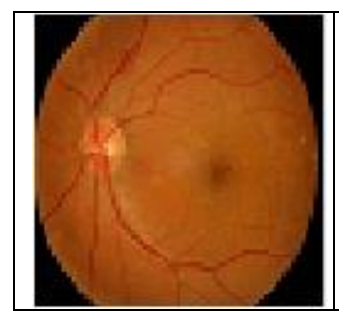

(a) original

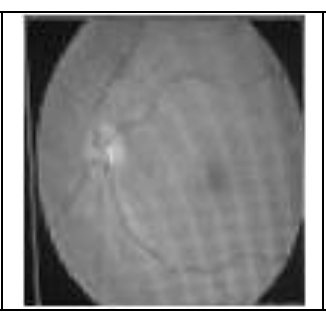

(b) gray level

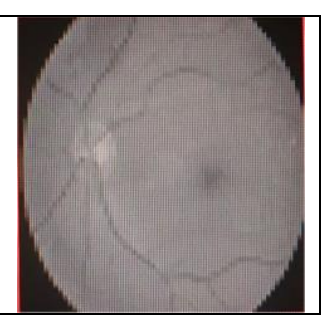

(c) enhanced image
Fig. 6. Hardware result for healthy image with $64 \times 64$ resolution

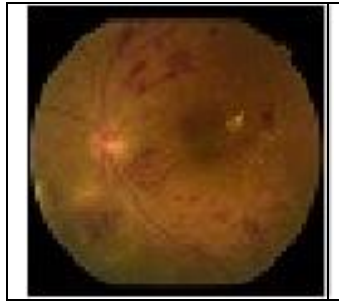

(a) original

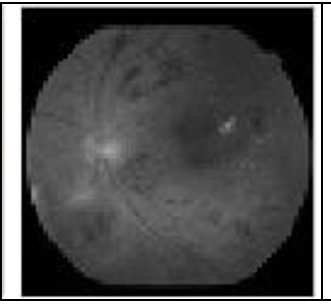

(b) gray level

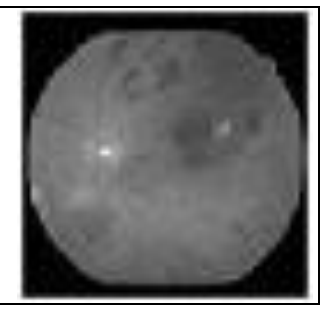

(c) enhanced image
Fig. 7. MatLab result for Moderate DR image with $64 \times 64$ resolution

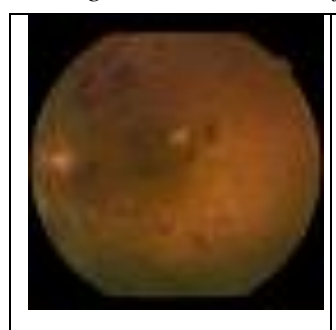

(a) original

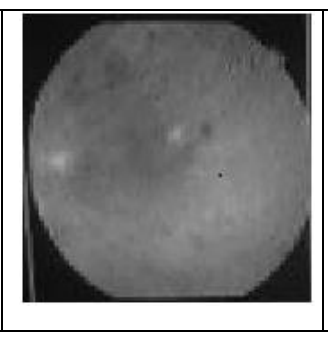

(b) gray level

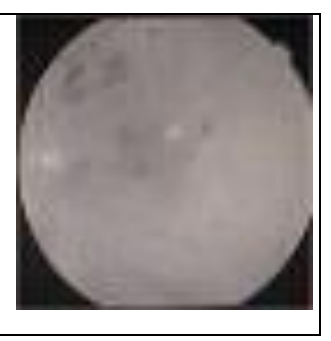

(c) enhanced image
Fig. 8. Hardware result for Moderate DR image with $64 \times 64$ resolution 


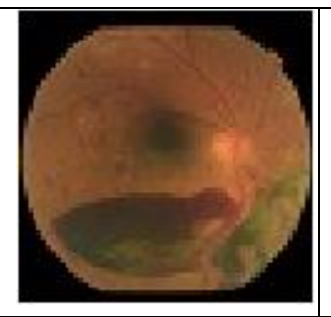

(a) original

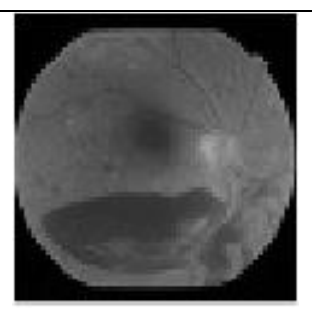

(b) gray level

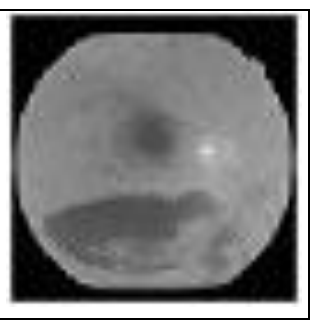

(c) enhanced image
Fig. 9. MatLab result for Severe DR image with $64 \times 64$ resolution

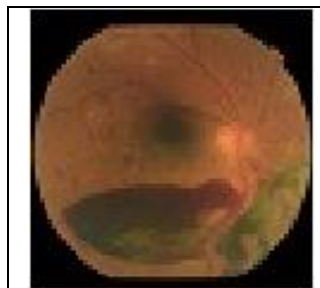

(a) original

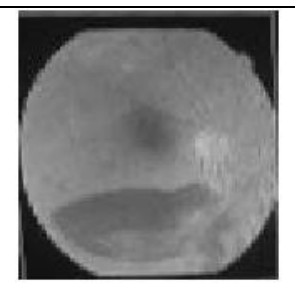

(b) gray level

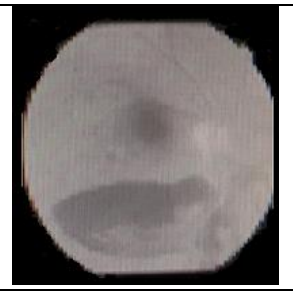

(c) enhanced image
Fig. 10. Hardware result for Severe DR image with $64 \times 64$ resolution

The execution time for this design is 612 faster than MATLAB time, while the overall maximaum frequency is equal to $72 \mathrm{MHz}$.

\section{CONCLUSIONS}

At the end of this work, it is possible to say that target of this work in designing and building a Fuzzy Histogram Equalization system using FPGA to enhance the contrast in diabetic retinopathy images as a first step in different diseases diagnosis has been achieved. The Fuzzy Histogram Equalization method can be used in medical fields like enhance the contrast in diabetic retinopathy images. The model is implemented and tested in MatLab for comparison purposes with the FPGA implementation which shows a slight difference between them due to the used platform limitation. The hardware platform (Spartan3E XC3S500E FPGA kit) has been used in this work to build the present design for Fuzzy Histogram Equalization system. Video Graphics Array (VGA) port is expanded sucsessfuly using expansion card then added to Fuzzy Histogram Equalization system to display input and output images on LCD screen to enhance their color resolution. The performance metrices AMBE, PSNR, MSE and ENTROPY is used for image performance measurments. System operating frequency is 72.521 $\mathrm{MHz}$, while the max occupation area reaches to $90 \%$. The speedup of operation of the $50 \mathrm{MHz}$ FPGA device on the $2.50 \mathrm{GHz}$ core i5 computer over the GPP (generalpurpose processor) program is 422 .

\section{REFERENCES}

[1] https://www.cs.tau.ac.il/ dcor/Graphics/cgslides/MATLAB-tutorial.pdf 29/12/2019.

[2] [2] J. Wolffsohn, (2008) "Eye essentials. Ophthalmic imaging", Edinburgh: Elsevier, Butterworth-Heinemann, pp. 1-172.

[3] [3] D B Younis and B MK Younis," Low Cost Histogram Implementation for Image Processing using FPGA", IOP Conf. Series: Materials Science and Engineering 745 (2020).

[4] [4] S. Sanchez-Solano, A. J. Cabrera, I. Baturone, F. J. Moreno- Velo, M. Brox, "FPGA implementation of embedded fuzzy controllers for robotic applications", IEEE Trans. Ind. Electron., vol. 54, no. 4, pp. 19371945, Aug. 2007.

[5] [5] Magudeeswaran V., \& Ravichandran C. G., "Fuzzy logic-based histogram equalization for image contrast enhancement", Mathematical Problems in Engineering, 2013, pp.1-10 (Article ID 891864), 2013.

[6] [6] Rahim SS, Palade V, Jayne C, Holzinger A, Shuttleworth J, "Detection of diabetic retinopathy and maculopathy in eye fundus images using fuzzy image processing", Springer, Heidelberg, Y. Guo et al. (Eds.): BIH 2015, vol. 9250, pp. 379-388, 2015. 